Supporting Information

\title{
Ultrasensitive Fiber-Based ZnO Nanowire Network Ultraviolet Photodetector Enabled by the Synergism between Interface and Surface Gating Effects
}

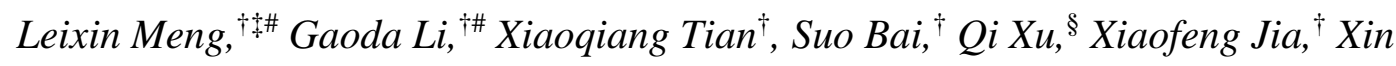

Cui, Yong Qin*广, and Wangsuo $W u^{\ddagger}$

${ }^{\dagger}$ Institute of Nanoscience and Nanotechnology and ${ }^{\ddagger}$ School of Nuclear Science and Technology, Lanzhou University, Lanzhou 730000, China

${ }^{\S}$ School of Advanced Materials and Nanotechnology, Xidian University, Xi’an 710071, China

" College of Chemistry and Chemical Engineering, Guangxi University, Guangxi 530004, China

\# L.M. and G.L. contributed equally to this work.

* Corresponding author email address: qinyong@lzu.edu.cn 


\section{CONTENTS}

Supporting Information Section 1: Characterization of $\mathrm{ZnO}$ microwires (MWs) and nanowires (NWs)

Supporting Information Section 2: Calculation of the current across a MW-MW or NW-NW junction

Supporting Information Section 3: KPFM detection of ZnO MW's surface barrier height change induced by UV light illumination

Supporting Information Section 4: Surface barrier height changes of $\mathrm{ZnO} \mathrm{NW}$ due to UV illumination

Supporting Information Section 5: Decrease of interface barrier height with the increase of applied voltage

Supporting Information Section 6: Fabrication of fiber-based $\mathrm{ZnO}$ nanowire network UV photodetector 
Supporting Information Section 1: Characterization of ZnO microwires (MWs) and nanowires (NWs)

ZnO MWs and NWs are synthesized simultaneously via chemical vapor deposition. The transparence of synthesized ZnO MWs (Figure S1a) implies MWs are single crystal. Thin plates of ZnO MW cut perpendicular to axial direction (Figure S1b, left) is characterized in transmission electron microscopy (TEM). Selected area electron diffraction (SAED) pattern (Figure S1b, middle) of the thin plate can be indexed as the [001] zone axis of single crystal $\mathrm{ZnO}$ with hexagonal structure, suggesting the single crystals structure grown along the [001] direction. The high-resolution TEM (HR-TEM) image (Figure S1b, middle) shows regular lattice with around $0.27 \mathrm{~nm}$ interplanar spacing, corresponding to $d$-spacing of (100) lattice planes of hexagonal ZnO. Both SAED and HR-TEM results indicate that the ZnO MWs are single crystal grown along the [001] direction.

ZnO NWs are also characterized with HR-TEM and SAED. Four different areas along a $\mathrm{ZnO} \mathrm{NW}$ shown in Figure S2a are selected and the corresponding HR-TEM images are shown in Figure S2b. All of them show a regular lattice with about $0.26 \mathrm{~nm}$ interplanar spacing, corresponding to the $d$-spacing of (002) lattice planes of hexagonal $\mathrm{ZnO}$. The results imply that the synthesized materials are probably single crystal ZnO NWs. It is then confirmed by SAED pattern shown in Figure S2c, which is indexed as the hexagonal wurtzite structure of $\mathrm{ZnO}$. 

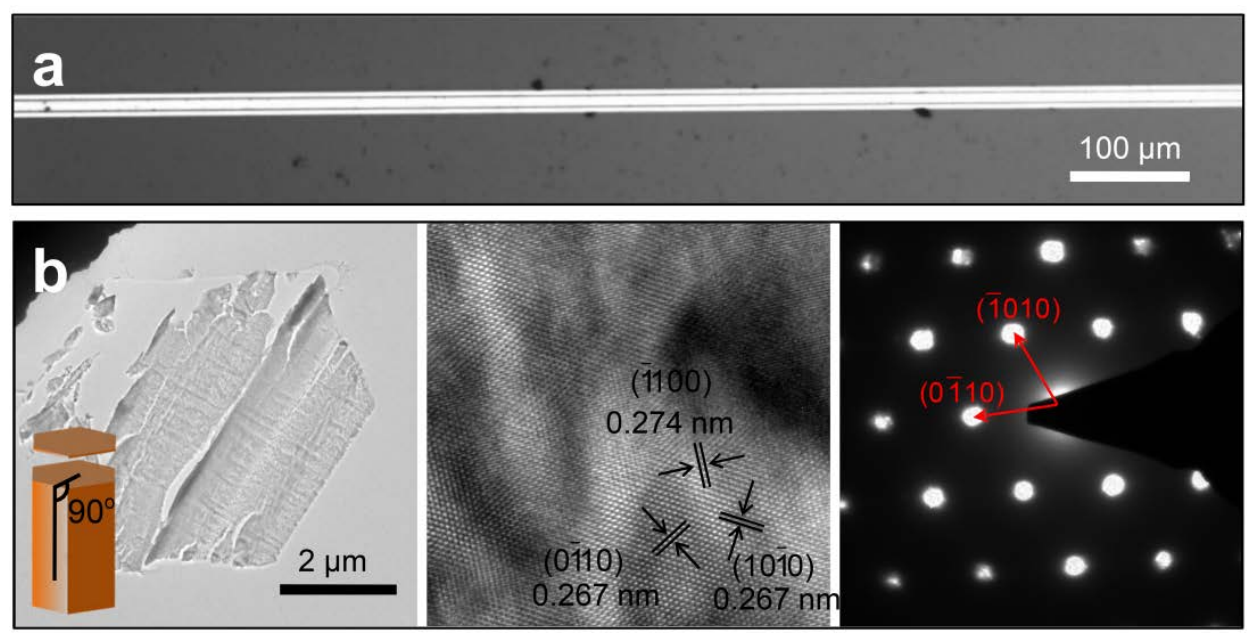

Figure S1. Structural characterization of ZnO MWs. (a) Typical optical images of a ZnO MW. (b) Left: TEM image of a thin plate cut from a ZnO MW, which is used for SAED and HRTEM characterizations. The inset illustrates the cut direction (perpendicular to the axial direction). Middle: HRTEM image of the thin plate shown in the left panel. Right: Corresponding SAED pattern.
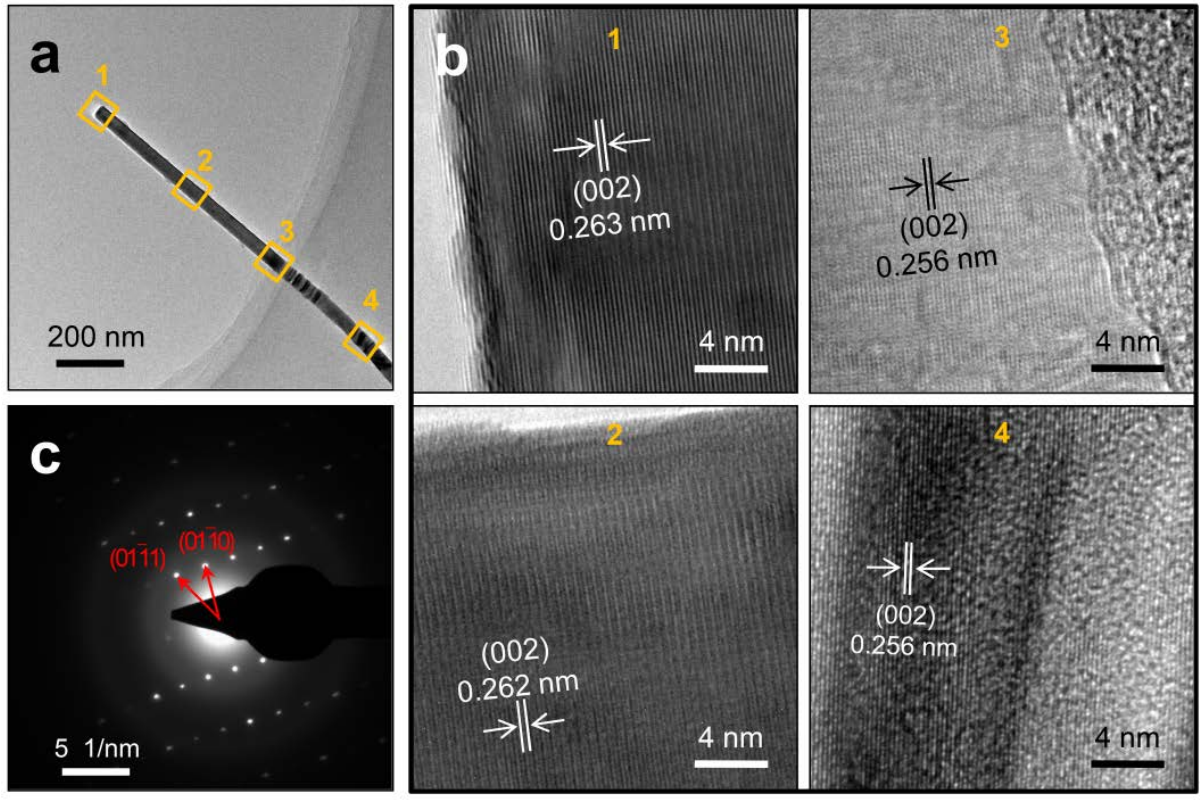

Figure S2. TEM characterization of ZnO NWs. a) Bright field TEM image of a ZnO

NW. The orange boxes indicate the positions for HR-TEM characterization. b)

Corresponding HR-TEM images indicated in (a). c) SAED pattern of the $\mathrm{ZnO} \mathrm{NW}$. 

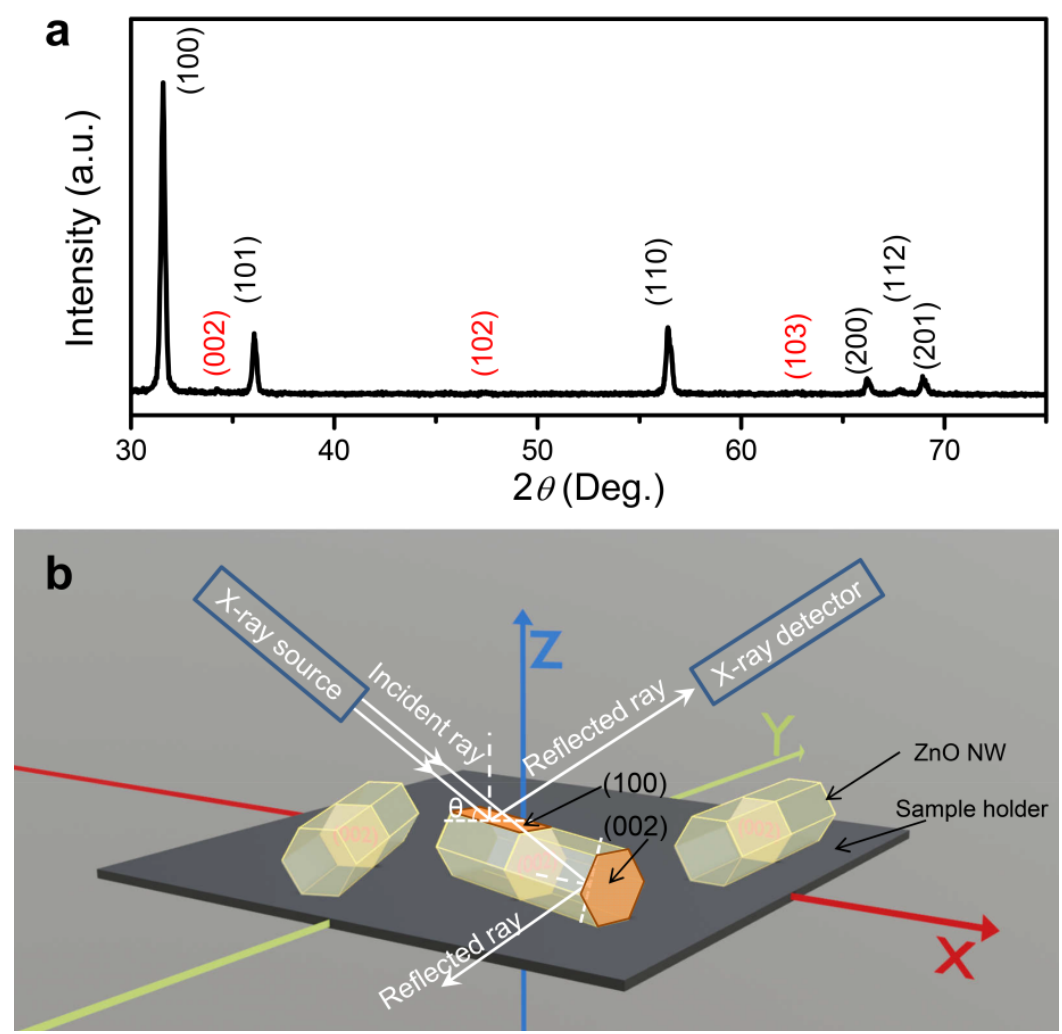

Figure S3 a) The XRD spectrum of ZnO NWs indicates ZnO NWs have hexagonal wurtzite structure. The peaks of (002), (102), and (103) planes are absent in the spectra. b) Schematic diagram of X-ray diffraction of ZnO NWs. The X-ray source and the X-ray detector scan in $\mathrm{X}-\mathrm{Z}$ plane.

X-ray powder diffraction (XRD) profile of the NWs is shown in Figure S3a. All peaks are assigned to $\mathrm{ZnO}$ with hexagonal wurtzite structure, which is consistent with the SAED result. Diffraction peaks of crystal face (002) and those nearly parallel to it, such as (102), and (103) are absent from the spectrum, as indicted by red texts. This is caused by the relative positions of $\mathrm{ZnO}$ (002) planes and the plane of incident X-ray and reflected X-ray to the detector: Since the plane of incident ray and reflected ray to X-ray detector, and the (002) plane are both perpendicular to the plane of sample holder (XY plane), no matter how the diffraction angle $\theta$ changes, the reflected ray 
from (002) plane can hardly reach the X-ray detector. As a result, (002) peak would be absent from the XRD spectra. This also holds true for the (102) and (103) peaks, which are nearly parallel with (002) plane. 
Supporting Information Section 2: Calculation of the current across a MW-MW or NW-NW junction

Based on the thermionic theory, when Schottky contact forms between a semiconductor and a metal, the current density formed by electron flow from semiconductor to metal can be written as: ${ }^{1}$

$$
J_{\mathrm{s} \rightarrow \mathrm{m}}=e n\left(\frac{k T}{2 \pi m_{\mathrm{n}}^{*}}\right)^{1 / 2} \exp \left[-\frac{e(\phi-V)}{k T}\right]
$$

where $e$ is the elementary charge, $n$ is the electron concentration at the conduction band minimum, $k$ is the Boltzmann constant, $T$ is the temperature, $m_{\mathrm{n}}^{*}$ is the effective mass of electrons at the bottom of conduction band, $e \phi$ is the barrier height, $V$ is the bias voltage applied at the two sides of the Schottky junction. When a positive voltage is applied to the metal, the barrier height would decrease, as shown in Figure S4.

a

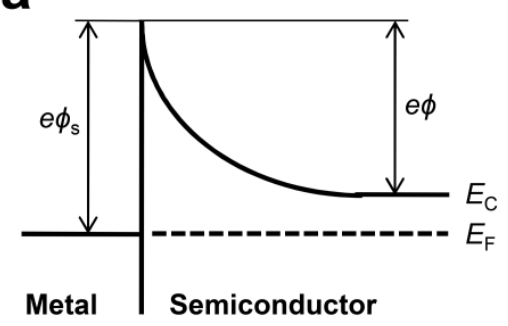

$(V=0)$ b

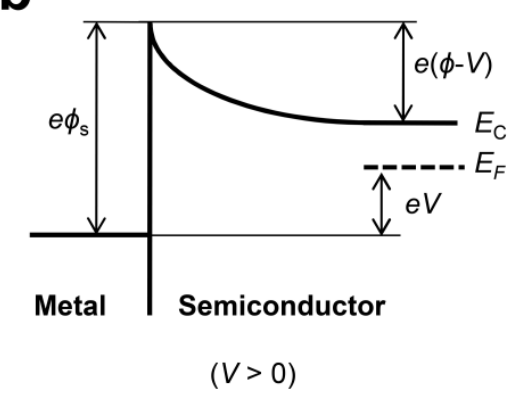

Figure S4. The influence of applied voltage on the band structure at metalsemiconductor interface. a) $V=0$, b) $V>0$.

A MW/NW junction is similar to a grain boundary, which can be simulated as two back to back Schottky junctions..$^{2-3}$ When a positive voltage is applied on Semiconductor \#2, the current density formed by electron flow from one semiconductor MW/NW to another can be written as: 


$$
J_{\mathrm{s} 1 \rightarrow \mathrm{s} 2}=e n\left(\frac{k T}{2 \pi m_{\mathrm{n}}^{*}}\right)^{1 / 2} \exp \left[-\frac{e\left(\phi-V_{1}\right)}{k T}\right]
$$

where $V_{1}$ is the potential decrease on the first MW/NW, and $V_{1}>0$. Note that electrons' thermionic emission also takes place in the reverse direction, forming a reverse current:

$$
J_{\mathrm{s} 2 \rightarrow \mathrm{s} 1}=-e n\left(\frac{k T}{2 \pi m_{\mathrm{n}}^{*}}\right)^{1 / 2} \exp \left[-\frac{e\left(\phi+V_{2}\right)}{k T}\right]
$$

$=V_{1}$

$+V_{2}$

Thus, the total current across the junction is:

$=J_{\mathrm{s} 1 \rightarrow \mathrm{s} 2}$

$+J_{\mathrm{s} 2 \rightarrow \mathrm{s} 1}$

By substituting Equation (S2), (S3) and (S4) into Equation (S5), the total current can be written as

$$
J=e n\left(\frac{k T}{2 \pi m_{\mathrm{n}}^{*}}\right)^{1 / 2} \exp \left(-\frac{e \phi}{k T}\right)\left\{\exp \left(\frac{e V_{1}}{k T}\right)-\exp \left[\frac{e\left(V_{1}-V\right)}{k T}\right]\right\}
$$

It should be noted that $\mathrm{ZnO} \mathrm{MWs} / \mathrm{NWs}$ ' conductivity $\left(\sigma_{\mathrm{MW}}\right)$ can be written as ${ }^{1}$ :

$=n e\left(\frac{e^{2} L^{2}}{2 k T \pi m_{n}^{*}}\right)^{1 / 2}$

where, $L$ is the MWs/NWs' channel length. So, ZnO MW junction photodetector's sensitivity can be written as the following Equation (Equation (1) in the main text): 


$$
J=\sigma_{\mathrm{MW}} \cdot \frac{k T}{e L} \exp \left(-\frac{e \phi}{k T}\right)\left\{\exp \left(\frac{e V_{1}}{k T}\right)\right.
$$

$$
\left.-\exp \left[\frac{e\left(V_{1}-V\right)}{k T}\right]\right\}
$$

a

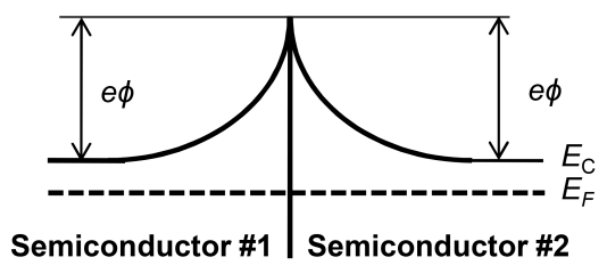

$(V=0)$ b

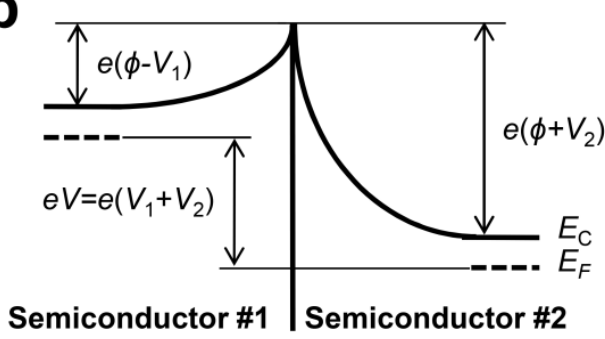

$(V>0)$

Figure S5. The influence of applied voltage on the band structure at semiconductorsemiconductor interface. a) $V=0$, b) $V>0$.

As discussed previously, ${ }^{3}$ for a polycrystalline film, an applied voltage would cause the depletion layer to increase considerably in the more positive grain but to decrease only slightly in the negative grain. This conclusion can also be used here: the voltage applied to the MW/NW junction UV photodetector mainly drops on semiconductor \#2 and $V_{1} \approx 0$. Thus, Equation (S6) is simplified as the following equation (Equation (2) in the main text):

$$
J=\sigma_{\mathrm{MW}} \cdot \frac{k T}{e L} \exp \left(-\frac{e \phi}{k T}\right)[1
$$

$$
\left.-\exp \left(-\frac{e V}{k T}\right)\right]
$$

In Equation (S9), both $\sigma_{\mathrm{MW}}$ and $\phi$ will change under UV illumination, so the sensitivity of the MW/NW junction UV photodetector can be written as:

$$
=\frac{\sigma_{\mathrm{MW}, \mathrm{UV}}}{\sigma_{\mathrm{MW}, \mathrm{dark}}} \cdot \exp \left(-\frac{e \Delta \phi}{k T}\right)-1,
$$

$$
S=\frac{J_{\mathrm{UV}}-J_{\mathrm{dark}}}{J_{\mathrm{dark}}}
$$


If the voltage has a very small value ( $\mathrm{eV}<<k T$ ), Equation (S9) can be written as Equation (S11) by using the linear approximation of $\exp (-e V / k T)$.

$$
J=e n \frac{e}{\left(2 \pi m_{\mathrm{n}}^{*} k T\right)^{1 / 2}} \exp \left(-\frac{e \phi}{k T}\right) V,
$$

Thus, if the applied voltage is small enough, the current and the voltage have a linear relation. 
Supporting Information Section 3: KPFM detection of ZnO MW's surface barrier height change induced by UV light illumination

We conducted Kelvin probe force microscopy (KPFM) measurement on a $\mathrm{ZnO} \mathrm{MW}$ to deduce the amount of the surface barrier height modulated by UV light (Figure S6a). The measurement was conducted on an atomic force microscopy (Dimension Icon, Bruker), using amplitude modulation mode and a MESP probe (Co/Cr coated). The ZnO MW was fixed on conductive carbon tape. ZnO’s surface barrier height $(e \phi)$ has the following relationship with the contact potential difference between the tip of the probe and $\mathrm{ZnO}(C P D)$ :

$$
e C P D=W_{\mathrm{m}}-\left[\chi+e \phi+E_{\mathrm{C}}\right.
$$

$$
\left.-\left(E_{\mathrm{F}}\right)_{\mathrm{ZnO}}\right]
$$

where, $W_{\mathrm{m}}$ is the work function of the tip, $\chi, E_{\mathrm{C}}$ and $\left(E_{\mathrm{F}}\right)_{\mathrm{Zno}}$ are $\mathrm{ZnO}$ 's electron affinity, conduction band level and Fermi level, respectively. In Equation (S12), CPD and $e \phi$ are UV light sensitive, while $W_{\text {tip }}, \chi$, and $E_{\mathrm{C}}-\left(E_{\mathrm{F}}\right)_{\mathrm{Zno}}$ don't change with UV light. So, the change of ZnO's surface barrier height ( $\left.e \Delta \phi=e \phi_{\mathrm{UV}}-e \phi_{\mathrm{dark}}\right)$ caused by UV light illumination is:

$=-e \triangle C P D$,

where $\triangle C P D\left(\triangle C P D=C P D_{\mathrm{UV}}-C P D_{\mathrm{dark}}\right)$ represents the change of $C P D$ under UV light illumination.

We measured the $C P D$ between the tip of the probe and the upper surface of the MW (the indicated position in the left panel of Figure S6a). The measurement is conducted 
in the dark at first. After measuring for $3 \mathrm{~min}$, UV LED $\left(\lambda_{\text {center }}=365 \mathrm{~nm}\right)$ is used to illuminate the MW. CPD immediately increases (Figure S6b). After another $8 \mathrm{~min}$, the UV LED is turned off, and $C P D$ decreased slowly. The value of $C P D$ 's increase under UV light can be deduced from Figure S6c, which is about $340 \mathrm{mV}$.
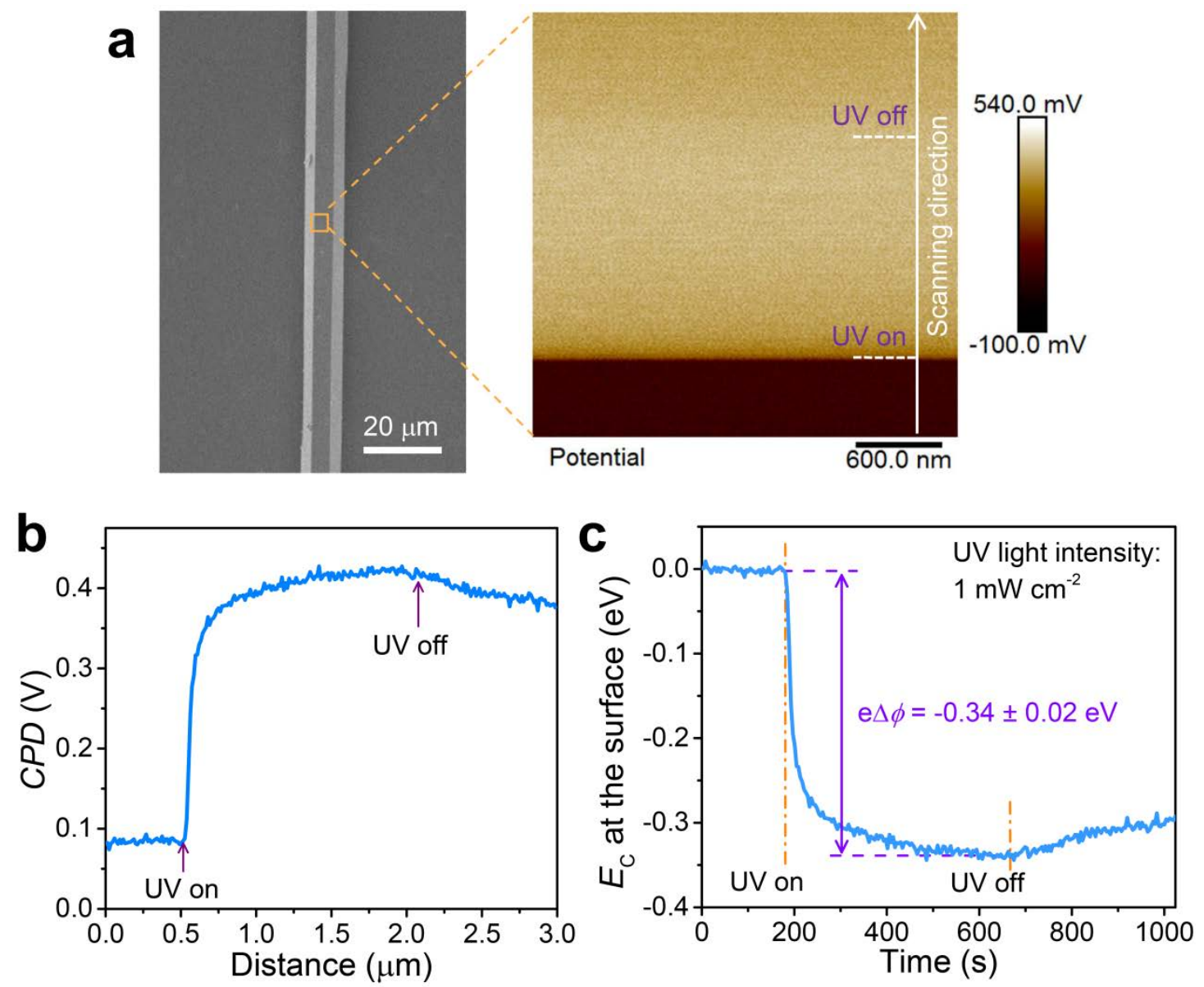

Figure S6. KPFM measurement results of a ZnO MW. a) SEM image and corresponding KPFM potential image of the indicated area. $1.0 \mathrm{~mW} \mathrm{~cm}{ }^{-2} \mathrm{UV}$ light was introduced at the 3th min and removed at the 11th min during the KPFM measurement. b) $C P D$ profile along the line shown in the right panel of (a). c) The change of ZnO's work function is measured by KPFM under UV light illumination with $1.0 \mathrm{~mW} \mathrm{~cm}^{-2}$ intensity. 
Supporting Information Section 4: Surface barrier height changes of ZnO NW due to UV illumination

When measuring the contact potential difference between small sample (such as $\mathrm{ZnO}$ NW) and the tip of AFM probe, the stray capacitance between components (tip, cantilever, substrate, and the sample) during the KPFM detection has a strong influence on the measurement. ${ }^{4}$ The contact potential difference between the sample and the tip is weighted by that between substrate and tip, as described in Equation (S14):

$$
\gamma C P \mathrm{D}_{\text {substrate }}+(1-\gamma) C P D_{\text {sample }}
$$

$$
=C P D_{\text {sample on substrate }}
$$

where $\gamma$ is the weight of the substrate. The weight of the substrate depends on the capacitance between different components (probe, sample and substrate) in measurement. Under UV light illumination, the change of $e \phi$ can be calculated based on Equation (S14):

$e \Delta \phi=-e \Delta C P D_{\mathrm{ZnO}}$

$=-\frac{e \Delta C P D_{\mathrm{ZnO} \text { on } \mathrm{Au}}}{1-\gamma}$

Our previous work described a calibrated method by conducting KPFM detection on ZnO NWs with a same diameter but on different substrates ( $\mathrm{Au}$ and $\mathrm{Cr}$ film sputtered on Si). ${ }^{4}$ Due to the capacitance between different components only depends on the geometric configuration, the weight of $\mathrm{Au}$ and $\mathrm{Cr}$ substrates are the same. By detecting ZnO NWs' CPDs on the two substrates, the weight can be quantified by taking Equation (S14) into consideration: 
$=\frac{C P D_{\mathrm{ZnO} \text { on } \mathrm{Au}}-C P D_{\mathrm{ZnO}} \text { on } \mathrm{Cr}}{C P D_{\mathrm{Au}}-C P D_{\mathrm{Cr}}}$

$C P D_{\mathrm{Au}}, C P D_{\mathrm{Cr}}, C P D_{\mathrm{Zno}}$ on $\mathrm{Au}$ and $C P D_{\mathrm{Zno} \text { on } \mathrm{Cr}}$ are measured contact potential differences of $\mathrm{Au}$ film, $\mathrm{Cr}$ film, $\mathrm{ZnO}$ on $\mathrm{Au}$ film, and $\mathrm{ZnO}$ on $\mathrm{Cr}$ film, respectively. So the surface barrier height changes of $\mathrm{ZnO}$ NW due to UV light illumination can be calculated based on Equation (S15) and (S16).

Experimentally, two ZnO NWs with diameters of $66 \mathrm{~nm}$ and $63 \mathrm{~nm}$ are put onto $\mathrm{Au}$ and Cr films, respectively (Figure S7a, b and c).

Potential profiles of the ZnO NWs over Au and Cr films (the green line in Figure S7d and the red line in Figure S7e, respectively) are shown in Figure S7f. They increase only 62.6 and $65.2 \mathrm{mV}$ upon UV light illumination respectively, which are around $18 \%$ of that of $\mathrm{ZnO}$ MW's (Figure S6d), implying the existence of large stray capacitances between components and the necessity to get the value of $\gamma$. Statistical results from Figure S7d and S7e are shown in Table S1 and $\gamma$ is calculated as 0.887. The change of work function is calculated based on the values shown in Figure S7f (red curve) and $\gamma$, and the results is plotted in Figure 2c of the main text. Surface barrier height change of the $\mathrm{ZnO}$ NWs due to UV light illumination $(e \Delta \phi)$ is calculated as $0.55 \pm 0.08 \mathrm{eV}$.

Table S1. KPFM measurement results of ZnO NWs.

\begin{tabular}{lll}
\hline & $C P D$ on $\mathrm{Au}(\mathrm{mV})$ & CPD on $\mathrm{Cr}(\mathrm{mV})$ \\
\hline Substrate & $-501.6 \pm 7.3$ & $7.4 \pm 5.7$ \\
ZnO in dark & $-453.8 \pm 8.5$ & $1.1 \pm 6.0$
\end{tabular}



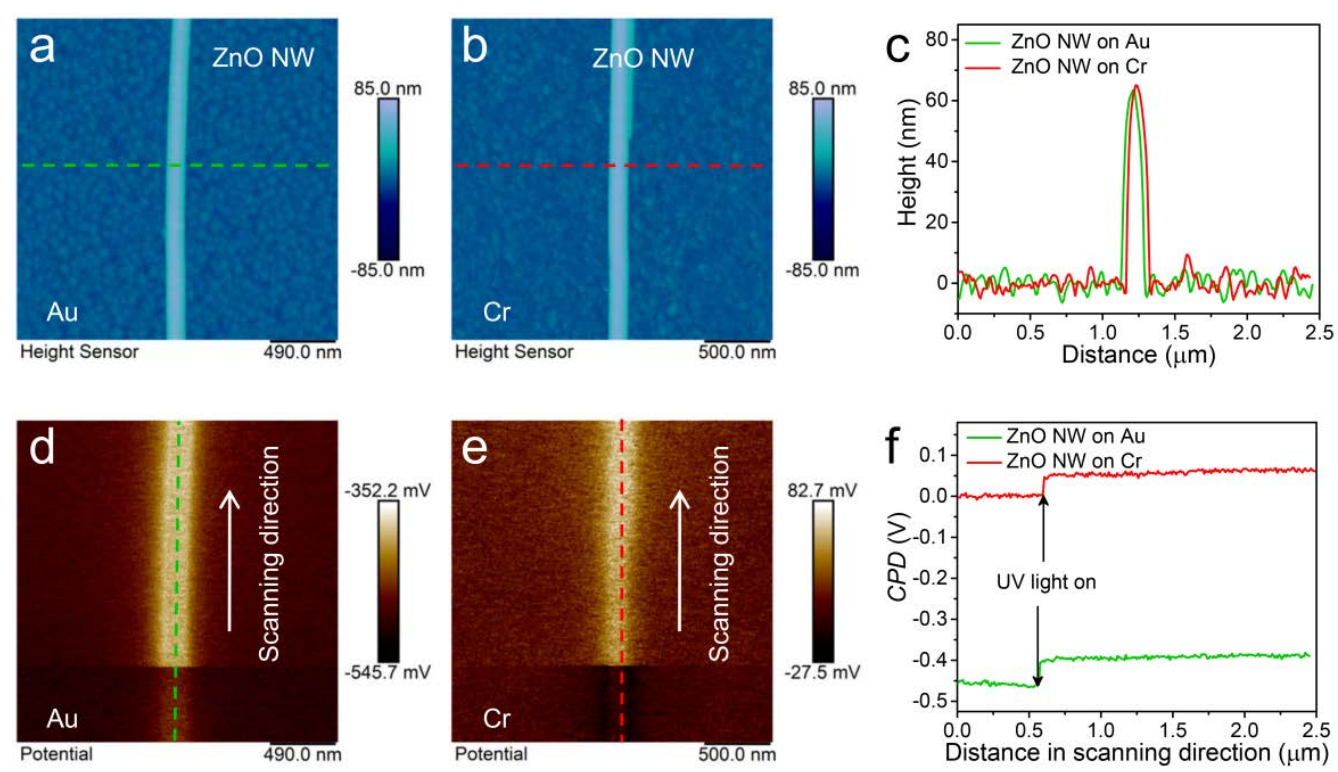

Figure S7. KPFM measurement results of ZnO NWs on Au and Cr. AFM images of the $\mathrm{ZnO} \mathrm{NW}$ on a) $\mathrm{Au}$ and b) Cr. c) The height profiles along the dashed lines in (a) and (b). CPDs of $\mathrm{ZnO}$ nanowires on d) $\mathrm{Au}$ and e) Cr. f) Potential profiles along the dashed lines in (d) and (e). 
Supporting Information Section 5: Decrease of interface barrier height with the increase of applied voltage

The relationship between interface barrier height and the applied voltage has been studied previously. ${ }^{3}$ Under a high applied voltage, the interface barrier height would decrease. However, due to the small current and large noise, it's quite hard to observe this phenomenon directly in ZnO NW junction UV photodetector. Consequently, ZnO MW junction is used to demonstrate this phenomenon. As shown in Figure S8a, the dark and UV photocurrents of ZnO MW and MW junction are compared. Since same electrodes are used in MW and MW junction UV photodetectors, the influence of metal-semiconductor barrier can be omitted because of the good linearity between the current and voltage of $\mathrm{ZnO} \mathrm{MW}$. As to the $\mathrm{ZnO} \mathrm{MW}$ junction, a dramatic increase of dark current is observed when the applied voltage is larger than $1.5 \mathrm{~V}$, which indicates a dramatic decrease of interface barrier height due to the large applied voltage. Consequently, the on/off ratio also decreases dramatically as shown in Figure S8b.
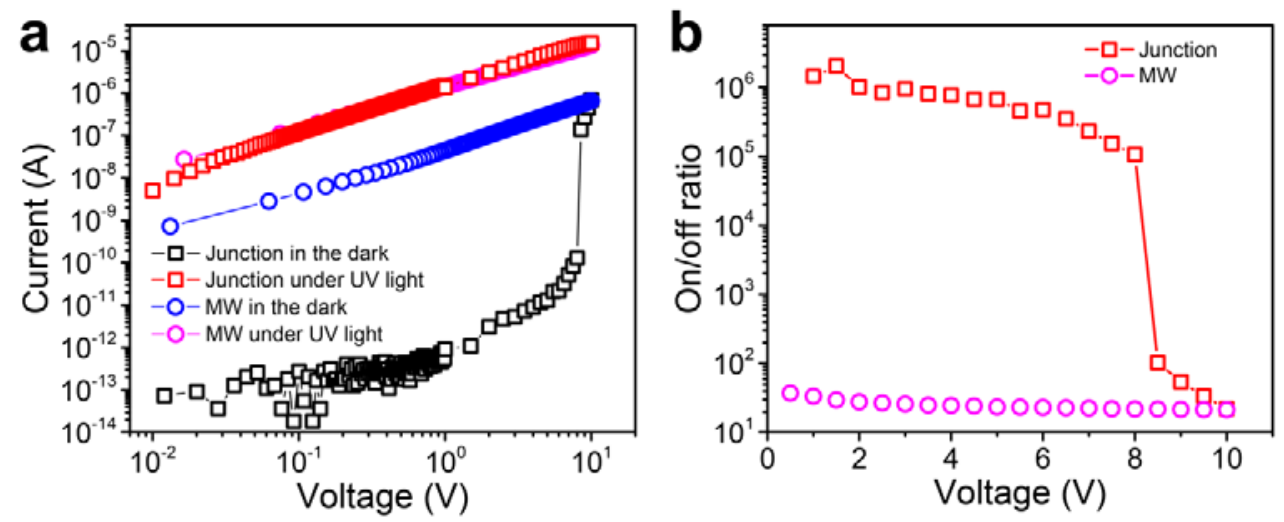

Figure S8. a) Dark and UV currents of ZnO MW and MW junction UV photodetectors. b) Relationship between on/off ratio and applied voltage of ZnO MW and MW junction UV photodetectors. 
Supporting Information Section 6: Fabrication of fiber-based $\mathrm{ZnO}$ nanowire junction UV photodetector

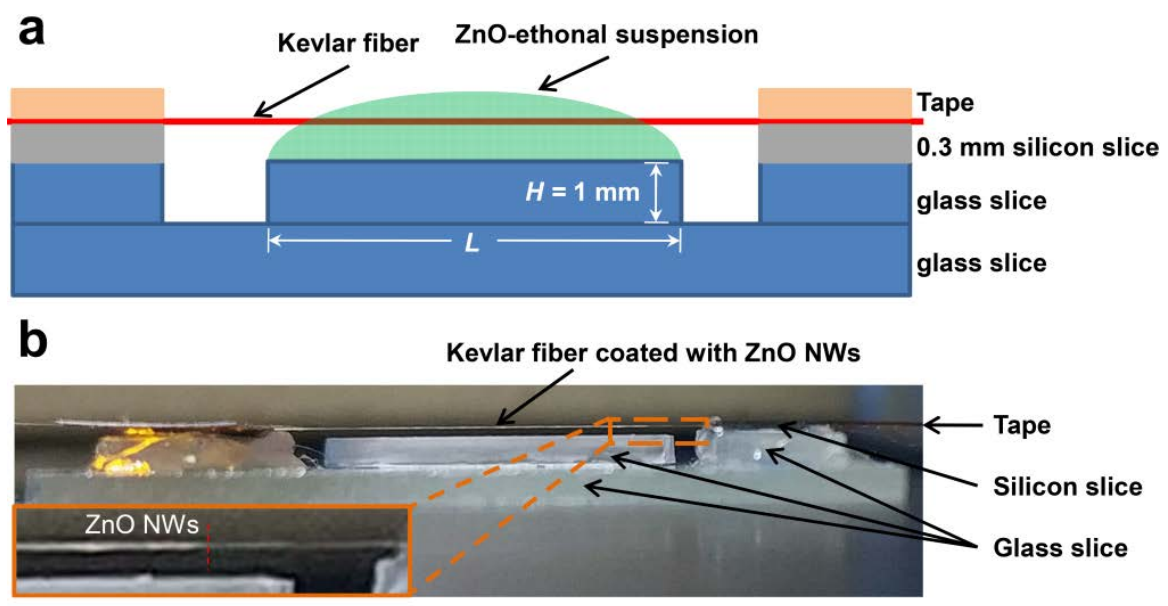

Figure S9. Experimental set-up for coating $\mathrm{ZnO} \mathrm{NWs}$ around Kevlar fiber. a) Schematic illustration of the set-up during the coating process. b) Photograph of the set-up after the coating process. The inset shows the zoom-in image of the indicated position, and the red dashed line shows the edge of the ZnO NWs coating.

\begin{tabular}{|lc|}
\hline $50 x$ & $500 \mu \mathrm{m}$ \\
\hline \hline $100 x$ & $200 \mu \mathrm{m}$ \\
\hline \hline $200 x$ & $100 \mu \mathrm{m}$ \\
\hline $500 x$ & $50 \mu \mathrm{m}$ \\
\hline $1000 x$ & \\
& \\
& \\
& \\
& \\
& \\
& \\
\hline
\end{tabular}

Figure S10. Photographs of a Kevlar fiber coated with ZnO NWs with different magnifications. 


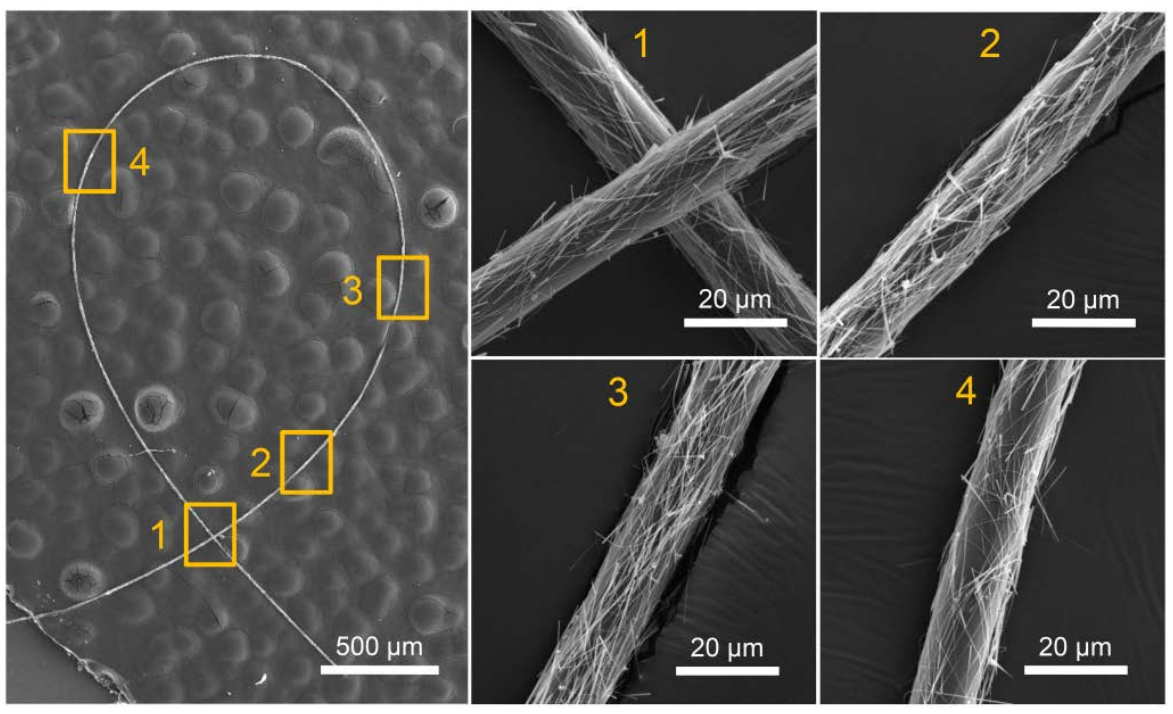

Figure S11. Fiber-based $\mathrm{ZnO}$ nanowire network is capable of curving in large degree and curvature, and the enlarged parts indicate that the curving or bending has no influence on its nanowire junction structure.

Table S2. Comparison of On/off ratio, response time $\left(\tau_{\mathrm{r}}\right)$, and decay time $\left(\tau_{\mathrm{d}}\right)$ of various UV photodetectors.

\begin{tabular}{cccccc}
\hline Materials & $\begin{array}{c}\text { Light } \\
\text { intensity } \\
\left(\mathrm{mW} \mathrm{cm}^{-2}\right)\end{array}$ & On/off ratio & $\tau_{\mathrm{r}}(\mathrm{s})$ & $\tau_{\mathrm{d}}(\mathrm{s})$ & Refs. \\
\hline $\begin{array}{c}\text { Fiber-based ZnO } \\
\text { nanowire-junction }\end{array}$ & 1.0 & $1.98 \times 10^{8}$ & 21.5 & 10.5 & This \\
$\begin{array}{c}\text { Cubic- } \\
\text { Zn } 0.51 \mathrm{Mg}_{0.49 O}\end{array}$ & 0.0019 & $1.48 \times 10^{7}$ & 163 & 44 & work \\
ZnO nanowire-Au & 1.3 & $10^{7}$ & - & - & 5 \\
ZnO nano particles & 0.1 & $3.4 \times 10^{5}$ & 250 & 150 & 7 \\
ZnO nano particles & 0.086 & $9.3 \times 10^{6}$ & 145 & 181 & 8 \\
ZnO nano particles & 0.080 & $1.9 \times 10^{7}$ & 5 & 9 & 9 \\
ZnO nano particles & 0.83 & $2.6 \times 10^{6}$ & - & - & 10 \\
ZnO polycrystalline & 0.1 & $1.3 \times 10^{6}$ & 62.6 & 30.1 & 11 \\
film & & $1.2 \times 10^{4}$ & 0.2 & 0.025 & 12 \\
p-NiO & $6.2 \times 10^{-4}$ & & & &
\end{tabular}


nanofilm

\begin{tabular}{cccccc} 
ZnO nanowires & 0.13 & $1.1 \times 10^{6}$ & - & 35.5 & 13 \\
$\mathrm{In}_{2} \mathrm{Ge}_{2} \mathrm{O}_{7}$ nanobelt & 0.019 & $10^{6}$ & $<0.3$ & $<0.3$ & 14 \\
$\mathrm{SnO}_{2}$ nanowires & 0.03 & $10^{7}\left(V_{\mathrm{g}}=-40\right.$ & & & 10 \\
$\mathrm{TiO}_{2}$ films & 2.44 & $10^{6}$ & $<0.5$ & $<0.5$ & 16 \\
$\begin{array}{c}\text { Single Crystal A- } \\
\text { EHDTT }\end{array}$ & 0.0014 & $1.4 \times 10^{5}$ & - & - & 17 \\
\hline
\end{tabular}




\section{REFERENCES}

(1) Sze, S. M., Physics of Semiconductor Devices /2nd Edition. Wiley: 1981; p 38.

(2) Wang, X.; Liao, M.; Zhong, Y.; Zheng, J. Y.; Tian, W.; Zhai, T.; Zhi, C.; Ma, Y.; Yao, J.; Bando, Y.; Golberg, D. ZnO Hollow Spheres with Double-Yolk Egg Structure for High-Performance Photocatalysts and Photodetectors. Adv. Mater. 2012, 24, 3421-3425.

(3) Pike, G. E.; Seager, C. H. The DC Voltage Dependence of Semiconductor Grain-Boundary Resistance. J. Appl. Phys. 1979, 50, 3414-3422.

(4) Li, G.; Meng, L.; Zhu, X.; Gao, W.; Qin, Y.; Chen, L. Clarifying the High On/Off Ratio Mechanism of Nanowire UV Photodetector by Characterizing Surface Barrier Height. Nanoscale 2018, 10, 2242-2248.

(5) Fan, M.-M.; Liu, K.-W.; Chen, X.; Zhang, Z.-Z.; Li, B.-H.; Zhao, H.-F.; Shen, D.-Z. Realization of cubic ZnMgO photodetectors for UVB applications. J. Mater. Chem. C 2015, 3, 313-317.

(6) Liu, K.; Sakurai, M.; Liao, M.; Aono, M. Giant Improvement of the Performance of ZnO Nanowire Photodetectors by Au Nanoparticles. J. Mater. Chem. C 2010, 114, 19835-19839.

(7) Nasiri, N.; Bo, R.; Wang, F.; Fu, L.; Tricoli, A. Ultraporous Electron-Depleted ZnO Nanoparticle Networks for Highly Sensitive Portable Visible-Blind UV Photodetectors. Adv. Mater. 2015, 27, 4336-4343.

(8) Nasiri, N.; Bo, R.; Chen, H.; White, T.P.; Fu, L.; Tricoli, A. Structural Engineering of Nano-Grain Boundaries for Low-Voltage UV-Photodetectors with Gigantic Photo- to Dark-Current Ratios. Adv. Opt. Mater. 2016, 4, 1787-1795. 
(9) Jin, Y.; Wang, J.; Sun, B.; Blakesley, J.C.; Greenham, N.C. Solution-processed ultraviolet photodetedtors based on colloidal ZnO nanoparticles. Nano Lett. 2008, 8, 1649-1653.

(10) Li, G.; Meng, L.; Zhu, X.; Gao, W.; Qin, Y.; Chen, L. Clarifying the High On/Off Ratio Mechanism of Nanowire UV Photodetector by Characterizing Surface Barrier Height. Nanoscale 2018, 10, 2242-2248.

(11) Xu, Q.; Cheng, L.; Meng, L.; Wang, Z.; Bai, S.; Tian, X.; Jia, X.; Qin, Y. Flexible Self-Powered ZnO Film UV Sensor with a High Response. ACS Appl. Mater. Interfaces 2019, 11, 26127-26133.

(12) Tyagi, M.; Tomar, M.; Gupta, V. Fabrication of an efficient GLAD-assisted p-NiO nanorod/n-ZnO thin film heterojunction UV photodiode. J. Mater. Chem. C 2014, 2, 2387-2393.

(13) Dai, Z.; Wei, L.; Xu, D.; Zhang, Y.; Ultraviolet photoresponse of ZnO nanowire thin-film transistors. Physica E 2012, 44,: 1999-2004.

(14) Li, L.; Lee, P.S.; Yan, C.; Zhai, T.; Fang, X.; Liao, M.; Koide, Y.; Bando, Y.; Golberg, D. Ultrahigh-Performance Solar-Blind Photodetectors Based on Individual Single-crystalline $\mathrm{In}_{2} \mathrm{Ge}_{2} \mathrm{O}_{7}$ Nanobelts. Adv. Mater. 2010, 22, 5145-5149.

(15) Kim, D.; Shin, G.; Yoon, J.; Jang, D.; Lee, S.-J.; Zi, G.; Ha, J.S. High performance stretchable UV sensor arrays of $\mathrm{SnO}_{2}$ nanowires. Nanotechnology 2013, 24, 315502.

(16) Lee, W.-J.; Hon, M.-H. An ultraviolet photo-detector based on $\mathrm{TiO}_{2} /$ water solid-liquid heterojunction. Appl. Phys. Lett. 2011, 99, 251102. 
(17) Kim, K.H.; Bae, S.Y.; Kim, Y.S.; Hur, J.A.; Hoang, M.H.; Lee, T.W.; Cho, M.J.; Kim, Y.; Kim, M.; Jin, J.-I.; Kim, S.-J.; Lee, K.; Lee, S.J.; Choi, D.H. Highly photosensitive J-aggregated single-crystalline organic transistors. Adv. Mater. 2011, 23, 3095-3099. 